\title{
REGULARLY LOG-PERIODIC FUNCTIONS AND SOME APPLICATIONS
}

BY

\author{
PÉTER KEVEI* (SzEGED)
}

Dedicated to the memory of Gyula Pap

\begin{abstract}
We prove a Tauberian theorem for the Laplace-Stieltjes transform, a Karamata-type theorem, and a monotone density theorem in the framework of regularly log-periodic functions. We provide several applications of these results: for example, we prove that the tail of a nonnegative random variable is regularly log-periodic if and only if the same holds for its Laplace transform at 0 , and we determine the exact tail behavior of fixed points of certain smoothing transforms.
\end{abstract}

2020 Mathematics Subject Classification: Primary 44A10; Secondary 60E99.

Key words and phrases: regularly log-periodic functions, Tauberian theorem, Karamata theorem, monotone density theorem, smoothing transform, semistable laws, supercritical branching processes

\section{INTRODUCTION}

A function $f:[0, \infty) \rightarrow[0, \infty)$ is regularly log-periodic, written $f \in \mathcal{R} \mathcal{L}$ or $f \in$ $\mathcal{R} \mathcal{L}(p, r, \rho)$, if $f$ is measurable, there is a function $\ell$ slowly varying at infinity, real numbers $\rho \in \mathbb{R}, r>1$, and a positive logarithmically periodic function $p \in \mathcal{P}_{r}$, such that

$$
\lim _{n \rightarrow \infty} \frac{f\left(r^{n} z\right)}{\left(r^{n} z\right)^{\rho} \ell\left(r^{n} z\right)}=p(z), \quad z \in C_{p},
$$

where $C_{p}$ stands for the set of continuity points of $p$, and for $r>1$,

* This research was supported by the János Bolyai Research Scholarship of the Hungarian Academy of Sciences, by the NKFIH grant FK124141, and by the EU-funded Hungarian grant EFOP-3.6.1-16-2016-00008. 


$$
\begin{aligned}
\mathcal{P}_{r}=\{p:(0, \infty) \rightarrow(0, \infty): & \inf _{x \in[1, r]} p(x)>0, p \text { is bounded, } \\
& \text { right-continuous, and } p(x r)=p(x), \forall x>0\} .
\end{aligned}
$$

This function class is a natural and important extension of regularly varying functions, and it appears in different areas of theoretical and applied probability. This class arises in connection with various random fixed point equations, such as the smoothing transform and the perpetuity equation. Regularly log-periodic functions are the basic ingredients in the theory of semistable laws. The tail of the limiting random variable of a supercritical Galton-Watson process is also regularly log-periodic. These facts are spelled out in detail in Section 3 . Here we only mention some results for the perpetuity equation

$$
X \stackrel{\mathcal{D}}{=} A X+B,
$$

where $(A, B)$ and $X$ on the right-hand side are independent. Under appropriate assumptions, Grincevičius [20, Theorem 2] showed that the tail of the solution of (1.2) is regularly log-periodic with constant slowly varying function. Under similar assumptions the same asymptotic behavior was shown for the max-equation $X \stackrel{\mathcal{D}}{=} \max \{A X, B\}$, which corresponds to the maximum of perturbed random walks (see Iksanov [25, Theorem 1.3.8]). More generally, this type of tail behavior appears in implicit renewal theory in the arithmetic case (see Jelenković and Olvera-Cravioto [26, Theorem 3.7] and Kevei [27]). In general, functions of the form $p(x) e^{\lambda x}, \lambda \in \mathbb{R}$, where $p$ is a periodic function, are solutions of certain integrated Cauchy functional equations (see Lau and Rao [29]). For physical relevance of log-periodicity we refer to Sornette [35].

The name 'regularly log-periodic' comes from Buldygin and Pavlenkov [10], [11], where a function $f$ is called regularly log-periodic if

$$
f(x)=x^{\rho} \ell(x) p(x), \quad x>0,
$$

where $\ell, \rho$ and $r$ are as above, and $p \in \mathcal{P}_{r}$ is continuous; see also Definition 5.13 in the recent monograph on extensions of regular variation by Buldygin et al. [9]. Condition (1.3) is stronger than (1.1); see Subsection 2.1 for examples and properties. In the examples given above, the continuity does not necessarily hold, and this is the reason for the extension of the definition. Moreover, our main motivation comes from the studies of the St. Petersburg distribution, where the corresponding $p$ function is not continuous (see Example 3.1 at the end of Subsection 3.1).

In what follows, we assume that $U:[0, \infty) \rightarrow[0, \infty)$ is a nondecreasing rightcontinuous function, and

$$
\widehat{U}(s)=\int_{[0, \infty)} e^{-s x} \mathrm{~d} U(x)
$$

denotes its Laplace-Stieltjes transform. 
For $r>1$ we further introduce the sets of functions

$$
\begin{aligned}
\mathcal{P}_{r, \rho}=\{p: & (0, \infty) \rightarrow(0, \infty): p \in \mathcal{P}_{r}, \text { and } \\
& \left.x^{\rho} p(x) \text { is nondecreasing if } \rho \geqslant 0, \text { and nonincreasing if } \rho<0\right\} .
\end{aligned}
$$

In order to characterize the Laplace-Stieltjes transform of regularly log-periodic functions, for $r>1$ and $\rho \geqslant 0$ put

$$
\begin{aligned}
\mathcal{Q}_{r, \rho}=\left\{q:(0, \infty) \rightarrow(0, \infty): s^{-\rho} q(s)\right. & \text { is completely monotone } \\
& \text { and } q(s r)=q(s), \forall s>0\} .
\end{aligned}
$$

For $\rho=0$ both $\mathcal{P}_{r, 0}$ and $\mathcal{Q}_{r, 0}$ are the set of constant functions.

The aim of the present paper is to prove a Tauberian theorem for the LaplaceStieltjes transform, and Karamata-type theorems in the framework of regularly log-periodic functions. The ratio Tauberian theorem [8, Theorem 2.10.1], a general version of the Tauberian theorem for Laplace-Stieltjes transforms, holds for O-regularly varying functions (see Section 2 for the definition). The equivalence of the behavior of $U$ at infinity and $\widehat{U}$ at zero holds if and only if $U^{*}(\lambda)=$ $\limsup _{x \rightarrow \infty} U(\lambda x) / U(x)$ is continuous at 1. The latter condition for functions defined in (1.3) is equivalent to the continuity of $p$ (see Proposition 2.2). In particular, the discontinuity of $p$ is the reason that the ratio Tauberian theorem [8, Theorem 2.10.1] does not hold in this setup. However, in Theorem 2.1 below we do provide an equivalence between the tail behavior of a function and the behavior of its Laplace-Stieltjes transform at zero. Karamata theorems in the sense of Theorems 1.5.11 (direct half) and 1.6.1 (converse half) of Bingham, Goldie and Teugels [8] for functions satisfying (1.3) with continuous $p$ were proved by Buldygin and Pavlenkov [10], [11]. Their results and further properties are contained in [9. Chapter 6]. Here we extend these results.

Section 2 contains the main results of the paper. After some preliminaries, we first deal with a Tauberian theorem for the Laplace-Stieltjes transform, then we prove the direct half of the Karamata theorem, and a monotone density theorem. In Section 3 we give some applications. We prove that the tail of a nonnegative random variable is regularly log-periodic if and only if the same is true for its Laplace transform at 0 . Using this result we determine the tail behavior of fixed points of certain smoothing transforms. We re-prove, in a special case, a result by Watanabe and Yamamuro [38] for tails of semistable random variables. Finally, we spell out some related results on limits of supercritical branching processes.

\section{RESULTS}

2.1. Preliminaries. First we discuss the place of regularly log-periodic functions among well-known function classes, which are extensions of regularly varying functions (extended, pseudo-, and O-regularly varying functions). For the definitions and properties of these functions we refer to the monographs [8], [9]. 
In the following we always assume that $f:[0, \infty) \rightarrow[0, \infty)$ is measurable. For $\lambda>0$ let

$$
f^{*}(\lambda)=\limsup _{x \rightarrow \infty} \frac{f(\lambda x)}{f(x)}, \quad f_{*}(\lambda)=\liminf _{x \rightarrow \infty} \frac{f(\lambda x)}{f(x)} .
$$

A function $f$ is extended regularly varying ([8, Section 2.0.2], [9, Definition 4.34]) if for some constants $c, d \in \mathbb{R}$,

$$
\lambda^{d} \leqslant f_{*}(\lambda) \leqslant f^{*}(\lambda) \leqslant \lambda^{c}, \quad \lambda>1
$$

pseudo-regularly varying [9, Definition 3.16] if

$$
\limsup _{\lambda \rightarrow 1} f^{*}(\lambda)=1
$$

and $O$-regularly varying ([8, Section 2.0.2], [9, Definition 3.7]) if for all $\lambda>0$,

$$
0<f_{*}(\lambda) \leqslant f^{*}(\lambda)<\infty
$$

First we note that regularly log-periodic functions can be quite irregular.

EXAMPLE 2.1. Consider the function

$$
f(x)= \begin{cases}n & \text { if } x \in\left[\left(1+n^{-1}\right) 2^{n},\left(1+2 n^{-1}\right) 2^{n}\right], n \geqslant 2 \\ 1 & \text { otherwise }\end{cases}
$$

Then formula $(1.1)$ holds with $\ell(x) \equiv 1, \rho=0, r=2$, and $p(x) \equiv 1$. Indeed, $\lim _{n \rightarrow \infty} f\left(2^{n} x\right)=1$ for every $x>0$, but $f$ is not even bounded, and the exceptional intervals are large.

For monotone log-periodic functions the situation is not so bad. A function $f:[0, \infty) \rightarrow[0, \infty)$ is ultimately monotone if it is monotone (nondecreasing or nonincreasing) for large enough $x$.

Proposition 2.1. Let $f \in \mathcal{R} \mathcal{L}(p, r, \rho)$ be ultimately monotone. Then

$$
\limsup _{x \rightarrow \infty} \frac{f(x)}{x^{\rho} \ell(x)}<\infty,
$$

and $f$ is $O$-regularly varying.

Proof. Assume that $f$ is ultimately nondecreasing; the nonincreasing case follows the same way.

For $\rho<0$ the boundedness of $p$ implies $\lim _{x \rightarrow \infty} f(x)=0$, therefore necessarily $\rho \geqslant 0$. Suppose, contrary to our claim, that $f\left(x_{n}\right) /\left(x_{n}^{\rho} \ell\left(x_{n}\right)\right) \rightarrow \infty$ for some $x_{n} \uparrow \infty$. Write $x_{n}=r^{k_{n}} z_{n}$, where $z_{n} \in[1, r)$. By the Bolzano-Weierstrass 
theorem, we may assume that $z_{n} \rightarrow \lambda \in[1, r]$. With some $\lambda<\eta \in C_{p}$, for large enough $n$,

$$
\frac{f\left(r^{k_{n}} z_{n}\right)}{\left(r^{k_{n}} z_{n}\right)^{\rho} \ell\left(r^{k_{n}} z_{n}\right)} \leqslant \frac{f\left(r^{k_{n}} \eta\right)}{\left(r^{k_{n}}\right)^{\rho} \ell\left(r^{k_{n}} z_{n}\right)},
$$

and the upper bound tends to $\eta^{\rho} p(\eta)$ as $n \rightarrow \infty$, which is a contradiction. The O-regular variation follows from the boundedness and strict positivity of $p$.

For extended regular variation, and for continuity of $f^{*}$, stronger conditions are needed. In the next statement we consider the subclass of regularly log-periodic functions for which the representation (1.3) holds for a slowly varying function $\ell$ with $\rho \in \mathbb{R}, r>1$, and $p \in \mathcal{P}_{r}$.

Proposition 2.2. Assume that $f \in \mathcal{R} \mathcal{L}(p, r, \rho)$ satisfies (1.3). Then $f$ is

(i) pseudo-regularly varying if and only if $p$ is continuous;

(ii) extended regularly varying if and only if $p$ is Lipschitz on $[1, r]$;

(iii) regularly varying if and only if $p$ is constant.

Note that a logarithmically periodic function is globally Lipschitz if and only if it is constant.

Proof of Proposition 2.2. The logarithmic periodicity of $p$ implies

$$
f^{*}(\lambda)=\lambda^{\rho} \sup _{x \in[1, r]} \frac{p(\lambda x)}{p(x)},
$$

from which we see that $f^{*}$ is continuous at 1 if and only if $p$ is continuous. By [9, Proposition 3.21] this is equivalent to pseudo-regular variation (see also [9. Remark 5.69]).

We turn to (ii). First we show that if $p$ is a Lipschitz function on $[1, r]$ then, by logarithmic periodicity, it is a Lipschitz function on $[a, b]$ for any $0<a<b<\infty$. Clearly, it is enough to show this for $[a, b]=\left[1, r^{2}\right]$. Let $L$ be the Lipschitz constant on $[1, r]$. Then for $x, y \in\left[r, r^{2}\right]$ we have

$$
|p(x)-p(y)|=|p(x / r)-p(y / r)| \leqslant \frac{L}{r}|x-y|,
$$

while for $x \in\left[r, r^{2}\right]$ and $y \in[1, r]$,

$$
|p(x)-p(y)| \leqslant|p(x)-p(r)|+|p(r)-p(y)| \leqslant \frac{L}{r}|x-r|+L|r-y| \leqslant L|x-y|,
$$

proving the claim.

From (2.4) we see that condition (2.1) holds for $\lambda$ large, so we only have to check it as $\lambda \downarrow 1$. Let $\lambda \in(1, r)$. If the Lipschitz constant of $p$ on $\left[1, r^{2}\right]$ is $L$, then for $x \in[1, r]$ we have $p(\lambda x) \leqslant p(x)+L x(\lambda-1)$, thus

$$
\sup _{x \in[1, r]} \frac{p(\lambda x)}{p(x)} \leqslant 1+L(\lambda-1) \sup _{x \in[1, r]} \frac{x}{p(x)} \leqslant \lambda^{c-\rho}
$$


for some $c>0$. The proof of the lower bound is similar. For the converse, assume contrary to our claim that $p$ is not Lipschitz. Then there are sequences $\lambda_{n} \downarrow 1$ and $x_{n} \rightarrow x \in[1, r]$ such that

$$
\left|p\left(\lambda_{n} x_{n}\right)-p\left(x_{n}\right)\right| \geqslant n x_{n}\left(\lambda_{n}-1\right),
$$

so 2.1) cannot hold. Finally, (iii) is obvious.

The following lemma from [27] states that for continuous $p$ the conditions (1.1] and (1.3) are equivalent.

Lemma 2.1. Assume that $f \in \mathcal{R} \mathcal{L}(p, r, \rho)$ is monotone and $p \in \mathcal{P}_{r}$ is continuous. Then

$$
f(x) \sim x^{\rho} \ell(x) p(x) \quad \text { as } x \rightarrow \infty .
$$

However, the next example shows that if $p$ is not continuous then (1.1) and (1.3) are different even for nondecreasing continuous functions.

EXAMPLE 2.2. Consider the function

$$
f(x)= \begin{cases}2^{n}, & 2^{n}+1 \leqslant x \leqslant 2^{n+1}-1, n \geqslant 1, \\ 2^{n-1}\left[1+h\left(\frac{x-2^{n}+1}{2}\right)\right], & 2^{n}-1 \leqslant x \leqslant 2^{n}+1, n \geqslant 1,\end{cases}
$$

where $h:[0,1] \rightarrow[0,1]$ is a continuous increasing function such that $h(0)=0$ and $h(1)=1$. For any $z \in(1,2)$,

$$
\lim _{n \rightarrow \infty} \frac{f\left(z 2^{n}\right)}{z 2^{n}}=z^{-1}=2^{-\left\{\log _{2} z\right\}},
$$

with $\{\cdot\}$ standing for fractional part. That is, 1.1 holds with $\rho=1, \ell \equiv 1, r=2$, $p(z)=2^{-\left\{\log _{2} z\right\}}$. For $z=1$, i.e. for $z \notin C_{p}$,

$$
\lim _{n \rightarrow \infty} \frac{f\left(2^{n}\right)}{2^{n}}=\frac{1}{2}(1+h(1 / 2)) \neq p(1) .
$$

Moreover, changing $h$ to $h_{1}$ and $h_{2}$ for $n$ even and odd, respectively, it is easy to construct continuous, nondecreasing examples where the limit in (1.1) does not even exist for $z \notin C_{p}$.

2.2. Tauberian theorem for the Laplace transform. Recall (1.5) and (1.6). There is a natural correspondence between $\mathcal{P}_{r, \rho}$ and $\mathcal{Q}_{r, \rho}$.

Lemma 2.2. For $p \in \mathcal{P}_{r, \rho}$ with $\rho>0$, define the operator $\mathrm{A}_{r, \rho}=\mathrm{A}_{\rho}$ as

$$
\mathrm{A}_{\rho} p(s)=s^{\rho} \int_{[0, \infty)} e^{-s x} \mathrm{~d}\left(p(x) x^{\rho}\right) .
$$

Then $\mathrm{A}_{\rho}: \mathcal{P}_{r, \rho} \rightarrow \mathcal{Q}_{r, \rho}$ is one-to-one. 
Proof. It is clear from the definition that $\mathrm{A}_{\rho} p \in \mathcal{Q}_{r, \rho}$.

Conversely, let $q \in \mathcal{Q}_{r, \rho}$. Since $s^{-\rho} q(s)$ is completely monotone, there is a nondecreasing right-continuous function $g:[0, \infty) \rightarrow[0, \infty)$ with $g(0)=0$ such that

$$
s^{-\rho} q(s)=\int_{[0, \infty)} e^{-s x} \mathrm{~d} g(x) .
$$

To prove that $p(x):=x^{-\rho} g(x) \in \mathcal{P}_{r, \rho}$ we only have to show the logarithmic periodicity of $p$. Substituting $s \rightarrow r s$ in 2.6 and using $q(r s)=q(s)$ we obtain

$$
\int_{[0, \infty)} e^{-s x} \mathrm{~d} g(x)=\int_{[0, \infty)} e^{-s x} \mathrm{~d}\left[r^{\rho} g(x / r)\right] .
$$

Uniqueness of the Laplace-Stieltjes transform implies $g(x)=r^{\rho} g(x / r)$ for $x \in C_{g}$, so $p(x)=p(x / r)$ for $x \in C_{p}$. If two right-continuous functions agree at all but countably many points, then they agree everywhere.

In the following, $\ell$ stands for a slowly varying function, either at infinity or at zero. The set of slowly varying functions at infinity [zero] is denoted by $\mathcal{S} \mathcal{V}_{\infty}$ $\left[\mathcal{S} \mathcal{V}_{0}\right]$. Recall the definition $\widehat{U}(s)=\int_{[0, \infty)} e^{-s x} \mathrm{~d} U(x)$ from 1.4).

THEOREM 2.1. Let $U:[0, \infty) \rightarrow[0, \infty)$ be a nondecreasing right-continuous function, $\rho \geqslant 0, r>1$, and let $\ell \in \mathcal{S} \mathcal{V}_{\infty}$. Then the conditions

$$
\lim _{n \rightarrow \infty} \frac{U\left(r^{n} z\right)}{\left(r^{n} z\right)^{\rho} \ell\left(r^{n} z\right)}=p(z) \quad \text { for each } z \in C_{p}, \text { for some } p \in \mathcal{P}_{r}
$$

and

$$
\widehat{U}(s) \sim s^{-\rho} \ell(1 / s) q(s) \quad \text { as } s \downarrow 0, \text { for some } q \in \mathcal{P}_{r},
$$

are equivalent. In each case, necessarily $p \in \mathcal{P}_{r, \rho}, q \in \mathcal{Q}_{r, \rho}$, and $\mathrm{A}_{\rho} p=q$ for $\rho>0$, and $p=q$ for $\rho=0$.

Moreover, if $p$ is continuous, then 2.7) implies

$$
U(x) \sim x^{\rho} \ell(x) p(x) \quad \text { as } x \rightarrow \infty .
$$

REMARK 2.1. (i) For $\rho=0$ the result follows from [8, Theorem 1.7.1].

(ii) The equivalence of $U\left(r^{n} z\right)=o\left(r^{n} \ell\left(r^{n}\right)\right)$ and $\widehat{U}(s)=o\left(s^{-\rho} \ell(1 / s)\right)$ also follows from [8, Theorem 1.7.1].

(iii) For continuous $p$ the ratio Tauberian theorem [8, Theorem 2.10.1] (see also Korenblyum [28], Feller [17], Stadtmüller and Trautner [36]) states that (2.8] and (2.9) are equivalent. Indeed, by Propositions 2.1 and 2.2 the function $U$ is always O-regularly varying, and $p$ is continuous if and only if $U^{*}(\lambda)$ is continuous at 1 . Moreover, the Laplace-Stieltjes transform of $x^{\rho} p(x)$ is $s^{-\rho} q(s)$. Theorem 2.10.1(iii) in [8] states that the continuity of $U^{*}$ at 1 is also necessary in general for the equivalence of (2.8) and (2.9). 
Proof of Theorem 2.1 In view of Remark2.1(i), we may assume that $\rho>0$. The proof follows the standard idea of Tauberian theorems (see [8, Theorem 1.7.1]) combined with Lemma2.1.

First we show the implication $2.7 \Rightarrow \Rightarrow 2.8$. The monotonicity of $U$ and 2.7 readily imply that $p \in \mathcal{P}_{r, \rho}$. From Proposition 2.1.

$$
\limsup _{x \rightarrow \infty} \frac{U(x)}{x^{\rho} \ell(x)}<\infty
$$

Using Potter's bounds we obtain, for $x$ large enough,

$$
\begin{aligned}
\widehat{U}\left(x^{-1}\right) & =\int_{[0, \infty)} e^{-y / x} \mathrm{~d} U(y) \leqslant U(x)+\sum_{n=1}^{\infty} e^{-2^{n-1}} U\left(2^{n} x\right) \\
& \leqslant 2 K x^{\rho} \ell(x)\left[1+\sum_{n=1}^{\infty} e^{-2^{n-1}} 2^{n(\rho+1)}\right],
\end{aligned}
$$

for some constant $K>0$. Therefore $\widehat{U}\left(x^{-1}\right) /\left(x^{\rho} \ell(x)\right)$ is bounded. Introduce the notation

$$
U_{x}(y)=\frac{U(x y)}{x^{\rho} \ell(x)}
$$

Using logarithmic periodicity, for any $z>0$ we have

$$
\lim _{n \rightarrow \infty} U_{r^{n}}(y)=y^{\rho} p(z y)=: V_{z}(y) \quad \text { for all } y \text { such that } z y \in C_{p} .
$$

It is easy to see that

$$
\widehat{U}_{x}(s)=\frac{\widehat{U}(s / x)}{x^{\rho} \ell(x)} .
$$

Since $U_{r^{n} z}(y)$ converges, the continuity and uniqueness theorem for LaplaceStieltjes transforms implies that

$$
\lim _{n \rightarrow \infty} \frac{\widehat{U}\left(s /\left(r^{n} z\right)\right)}{\left(r^{n} z\right)^{\rho} \ell\left(r^{n} z\right)}=\widehat{V}_{z}(s)
$$

for all $s>0$, as $\widehat{V}_{z}$, being a Laplace-Stieltjes transform, is continuous. Choosing $s=1$, after a short calculation we have

$$
\lim _{n \rightarrow \infty} \frac{\widehat{U}\left(1 /\left(r^{n} z\right)\right)}{\left(r^{n} z\right)^{\rho} \ell\left(r^{n} z\right)}=q(1 / z)
$$

with $q=\mathrm{A}_{\rho} p$. With the notation $f(x)=\widehat{U}(1 / x)$ we obtain

$$
\lim _{n \rightarrow \infty} \frac{f\left(r^{n} z\right)}{\left(r^{n} z\right)^{\rho} \ell\left(r^{n} z\right)}=q(1 / z)
$$


for each $z>0$, since $q$ is continuous. Lemma 2.1 implies $f(x) \sim x^{\rho} \ell(x) q(1 / x)$ as $x \rightarrow \infty$, which is the same as $\widehat{U}(s) \sim s^{-\rho} \ell(1 / s) q(s)$ as $s \downarrow 0$, as stated.

Next we show the implication $(2.8) \Rightarrow(2.7)$. Note that $(2.8)$ implies

$$
\widehat{U}_{x}(s)=\frac{\widehat{U}(s / x)}{x^{\rho \ell}(x)} \sim s^{-\rho} q(s / x) \quad \text { as } x \rightarrow \infty .
$$

Since $q \in \mathcal{P}_{r}$ we have, for any $z>0$,

$$
\lim _{n \rightarrow \infty} \widehat{U}_{r^{n} z}(s)=s^{-\rho} q(s / z) .
$$

Therefore, the continuity theorem gives

$$
\lim _{n \rightarrow \infty} U_{r^{n} z}(y)=u_{z}(y), \quad y \in C_{u_{z}},
$$

for some nondecreasing function $u_{z}$. Therefore $\widehat{u}_{z}(s)=s^{-\rho} q(s / z)$, which implies $q \in \mathcal{Q}_{r, \rho}$. A short calculation shows that the right-hand side of (2.11) is the Laplace-Stieltjes transform of $u_{z}(y):=y^{\rho} p(z y)$. Note that $1 \in C_{u_{z}}$ whenever $z \in C_{p}$, and thus 2.7 holds.

The last statement follows from Lemma 2.1 .

The same proof gives an analogous result in the case $x \downarrow 0, s \rightarrow \infty$ (see [8, Theorem 1.7.1']).

2.3. Karamata and monotone density theorems. Let $\mathcal{P}_{r, \rho}^{m}$ denote the set of functions in $\mathcal{P}_{r, \rho}$ which are $m$ times differentiable on $(0, \infty)$ (we do not assume continuity of the $m$ th derivative). For $r>1$ and $\rho>0$ introduce the operator $\mathrm{B}_{r, \rho}=\mathrm{B}_{\rho}: \mathcal{P}_{r} \rightarrow \mathcal{P}_{r, \rho}^{1}$ by setting

$$
\mathrm{B}_{\rho} p(x)=x^{-\rho} \int_{0}^{x} y^{\rho-1} p(y) \mathrm{d} y .
$$

A short calculation using logarithmic periodicity shows that

$$
\int_{0}^{r^{m}} s^{\rho-1} p(s) \mathrm{d} s=\frac{r^{m \rho}}{r^{\rho}-1} \int_{1}^{r} s^{\rho-1} p(s) \mathrm{d} s,
$$

and thus

$$
\mathrm{B}_{\rho} p(x)=r^{-\rho\left\{\log _{r} x\right\}}\left[\frac{1}{r^{\rho}-1} \int_{1}^{r} s^{\rho-1} p(s) \mathrm{d} s+\int_{1}^{r^{\left\{\log _{r} x\right\}}} s^{\rho-1} p(s) \mathrm{d} s\right],
$$

where $\{x\}=x-\lfloor x\rfloor$ stands for the fractional part of $x$. It is easy to see that $\mathrm{B}_{\rho} p \in \mathcal{P}_{r, \rho}^{1}$. Moreover, it is one-to-one with inverse

$$
\mathrm{B}_{\rho}^{-1} q(x)=x^{1-\rho} \frac{\mathrm{d}}{\mathrm{d} x}\left[x^{\rho} q(x)\right], \quad q \in \mathcal{P}_{r, \rho}^{1} .
$$

The following statement is a Karamata-type theorem for regularly log-periodic functions (see [8, Theorem 1.5.11]). 
THEOREM 2.2. Assume that for some $\rho>0$,

(2.15) $\lim _{n \rightarrow \infty} \frac{u\left(r^{n} z\right)}{\left(r^{n} z\right)^{\rho-1} \ell\left(r^{n} z\right)}=p_{0}(z) \quad$ for each $z \in C_{p_{0}}$, for some $p_{0} \in \mathcal{P}_{r}$, $u$ is locally integrable, and

$$
\limsup _{x \rightarrow \infty} \frac{u(x)}{x^{\rho-1} \ell(x)}<\infty .
$$

Then, with $p=\mathrm{B}_{\rho} p_{0}$,

$$
U(x)=\int_{0}^{x} u(y) \mathrm{d} y \sim x^{\rho} \ell(x) p(x) \quad \text { as } x \rightarrow \infty .
$$

REMARK 2.2. (i) For continuous $p_{0}$ the condition $u(x) \sim x^{\rho-1} \ell(x) p_{0}(x)$ as $x \rightarrow \infty$ implies 2.17) (see [11, Lemma 3] and [9, Theorem 6.6]; compare them to formula (2.13), noting that our $\rho$ and their $\rho$ are different.)

(ii) It is again straightforward to extend this result to the case when the limit in (2.15) is zero.

Proof of Theorem 2.2 From (2.16) we readily find as in [8, Proposition 1.5.8] that

$$
\limsup _{x \rightarrow \infty} \frac{U(x)}{x^{\rho} \ell(x)}<\infty
$$

A short calculation gives, for any $0<\varepsilon<1$,

$$
\frac{U\left(r^{n} z\right)-U\left(r^{n} z \varepsilon\right)}{\left(r^{n} z\right)^{\rho} \ell\left(r^{n} z\right)}=\int_{\varepsilon}^{1} \frac{u\left(r^{n} z t\right)}{\left(r^{n} z t\right)^{\rho-1} \ell\left(r^{n} z t\right)} t^{\rho-1} \frac{\ell\left(r^{n} z t\right)}{\ell\left(r^{n} z\right)} \mathrm{d} t .
$$

Whenever $z t \in C_{p}$ the integrand converges to $p_{0}(z t) t^{\rho-1}$. Since the set of discontinuity points of a right-continuous function is at most countable, and an integrable majorant exists by (2.16) and by an application of Potter's bounds, we see that

$$
\lim _{n \rightarrow \infty} \frac{U\left(r^{n} z\right)-U\left(r^{n} z \varepsilon\right)}{\left(r^{n} z\right)^{\rho} \ell\left(r^{n} z\right)}=\int_{\varepsilon}^{1} t^{\rho-1} p_{0}(z t) \mathrm{d} t .
$$

Finally, (2.18) implies

$$
\limsup _{\varepsilon \downarrow 0} \limsup _{n \rightarrow \infty} \frac{U\left(r^{n} z \varepsilon\right)}{\left(r^{n} z\right)^{\rho} \ell\left(r^{n} z\right)}=0 .
$$

Combining the last two limit relations we obtain

$$
\begin{aligned}
\lim _{n \rightarrow \infty} \frac{U\left(r^{n} z\right)}{\left(r^{n} z\right)^{\rho} \ell\left(r^{n} z\right)} & =\int_{0}^{1} t^{\rho-1} p_{0}(z t) \mathrm{d} t \\
& =z^{-\rho} \int_{0}^{z} s^{\rho-1} p_{0}(s) \mathrm{d} s=\mathrm{B}_{\rho} p_{0}(z) .
\end{aligned}
$$

Since $\mathrm{B}_{\rho} p_{0}$ is continuous, the statement follows from Lemma 2.1. 
The statement remains true for $\rho=0$ in the following version. For similar results for the more general class of regularly log-bounded functions see [11, Conjecture 1] and [9, Proposition 6.7]. Just as condition (2.16) in Theorem 2.2, condition (2.21) below is not very restrictive, and necessary in general.

Lemma 2.3. Assume that for some $p_{0} \in \mathcal{P}_{r}$,

$$
\lim _{n \rightarrow \infty} \frac{r^{n} z u\left(r^{n} z\right)}{\ell\left(r^{n} z\right)}=p_{0}(z) \quad \text { for each } z \in C_{p_{0}},
$$

$u$ is locally integrable, and

$$
0<\liminf _{x \rightarrow \infty} \frac{x u(x)}{\ell(x)} \leqslant \limsup _{x \rightarrow \infty} \frac{x u(x)}{\ell(x)}<\infty .
$$

Then $U(x)=\int_{0}^{x} u(y) \mathrm{d} y$ is slowly varying, and $\lim _{x \rightarrow \infty} U(x) / \ell(x)=\infty$.

Proof. The proof is similar to the proof of [8, Proposition 1.5.9a]. Put

$$
\liminf _{x \rightarrow \infty} \frac{x u(x)}{\ell(x)}=: k>0
$$

Then

$$
\liminf _{x \rightarrow \infty} \frac{U(x)}{\ell(x)} \geqslant \frac{k}{2} \liminf _{x \rightarrow \infty} \frac{1}{\ell(x)} \int_{\varepsilon x}^{x} \frac{\ell(y)}{y} \mathrm{~d} y=\frac{k}{2} \log \varepsilon^{-1} .
$$

As $\varepsilon \downarrow 0$ we get $\lim _{x \rightarrow \infty} U(x) / \ell(x)=\infty$. Put $\varepsilon(x)=x u(x) / U(x)$. We have shown that $\lim _{x \rightarrow \infty} \varepsilon(x)=0$. Noticing

$$
\frac{\mathrm{d}}{\mathrm{d} x} \log U(x)=\frac{U^{\prime}(x)}{U(x)}=\frac{\varepsilon(x)}{x},
$$

we see that the representation theorem for slowly varying functions $[8$, Theorem 1.3.1] implies the statement.

The converse part of Karamata's theorem for regularly log-periodic functions was proved in [11] (see also [9, Theorem 6.8]). Here we deal with monotone density results, which can also be seen as a converse of Theorem 2.2 .

THEOREM 2.3. Assume that $U(x)=\int_{0}^{x} u(y) \mathrm{d} y, u$ is ultimately monotone, and (2.7) holds with $\rho \geqslant 0$. If $\rho>0$, then $p=\mathrm{B}_{\rho} p_{0}$ for some $p_{0} \in \mathcal{P}_{r}$. For $\rho=0$ let $p_{0}(x) \equiv 0$. In both cases

$$
\lim _{n \rightarrow \infty} \frac{u\left(r^{n} z\right)}{\left(r^{n} z\right)^{\rho-1} \ell\left(r^{n} z\right)}=p_{0}(z) \quad \text { for each } z \in C_{p_{0}} .
$$

Moreover, if $p_{0}$ is continuous, then

$$
u(x) \sim x^{\rho-1} \ell(x) p_{0}(x) \quad \text { as } x \rightarrow \infty .
$$


REMARK 2.3. (i) We see from the statement that if (2.7) holds, and $U$ has an ultimately monotone density, then $p$ in 2.7 is necessarily differentiable.

(ii) For $\rho=0$ the statement follows from the 'usual' monotone density theorem [8, Theorem 1.7.2], since $p \in \mathcal{P}_{r}$ is necessarily constant. Theorem 1.7.2 in [8] also implies that the result remains true when the limit $p$ in (2.7) is zero.

Proof of Theorem 2.3. By (2.7),

$$
\frac{U(b x)-U(a x)}{x^{\rho} \ell(x)}=\int_{a}^{b} \frac{u(s x)}{x^{\rho-1} \ell(x)} \mathrm{d} s
$$

is bounded as $x \rightarrow \infty$. Since $u$ is ultimately monotone, this readily implies that the integrand is bounded too as $x \rightarrow \infty$, which allows us to use Helly's selection theorem. Fix $z>0$, and consider the sequence $r^{n} z$. By the selection theorem, there is a subsequence $n_{k}$ and a monotone limit function $v_{z}$ such that

$$
\lim _{k \rightarrow \infty} \frac{u\left(r^{n_{k}} z s\right)}{\left(r^{n_{k}} z\right)^{\rho-1} \ell\left(r^{n_{k}} z\right)}=v_{z}(s) \quad \text { for each } s \in C_{v_{z}} .
$$

On the other hand, $U(x y) /\left(x^{\rho} \ell(x)\right)$ converges on the sequence $r^{n} z$, and thus for the limit function $v_{z}$,

$$
\int_{a}^{b} v_{z}(s) \mathrm{d} s=b^{\rho} p(b z)-a^{\rho} p(a z)
$$

for $0<a<b<\infty$ such that $a z, b z \in C_{p}$. This clearly determines the limit function at its continuity points, and so the convergence in 2.22 holds along the whole sequence $n$. The latter implies that $v_{z}(r s)=r^{\rho-1} v_{z}(s)$. From 2.23 we find that $p \in \mathcal{P}_{r, \rho}^{1}$. Let $p_{0}=\mathrm{B}_{\rho}^{-1} p$. By 2.14 ,

$$
v_{z}(s)=\frac{\mathrm{d}}{\mathrm{d} s}\left(s^{\rho} p(s z)\right)=s^{\rho-1} p_{0}(s z) .
$$

If $z \in C_{p_{0}}$, then $s=1$ is a continuity point of $v_{z}$ in 2.22 , and the first statement follows. The second follows from Lemma 2.1.

The following statements are versions of the previous results, which we need later. Since the proofs are the same, we omit them.

First we deal with the case when $\rho<0$. As before, let $\mathcal{P}_{r, \rho}^{1}$ denote the set of functions in $\mathcal{P}_{r, \rho}$ which are differentiable on $(0, \infty)$. For $r>1$ and $\rho<0$ introduce the operator $\mathrm{B}_{r, \rho}=\mathrm{B}_{\rho}: \mathcal{P}_{r} \rightarrow \mathcal{P}_{r, \rho}^{1}$ by setting

$$
\mathrm{B}_{\rho} p(x)=x^{-\rho} \int_{x}^{\infty} y^{\rho-1} p(y) \mathrm{d} y .
$$

As before $\mathrm{B}_{\rho} p \in \mathcal{P}_{r, \rho}^{1}$, and $\mathrm{B}_{\rho}$ is one-to-one with inverse

$$
\mathrm{B}_{\rho}^{-1} q(x)=-x^{1-\rho} \frac{\mathrm{d}}{\mathrm{d} x}\left[x^{\rho} q(x)\right], \quad q \in \mathcal{P}_{r, \rho}^{1} .
$$


Proposition 2.3. Let $U(x)=\int_{x}^{\infty} u(y) \mathrm{d} y$, where $u$ is locally integrable, $r>1$, and $\rho<0$. If $\lim \sup _{x \rightarrow \infty} \frac{u(x)}{x^{\rho-1} \ell(x)}<\infty$ then the condition

$$
\lim _{n \rightarrow \infty} \frac{u\left(r^{n} z\right)}{\left(r^{n} z\right)^{\rho-1} \ell\left(r^{n} z\right)}=p_{0}(z) \quad \text { for each } z \in C_{p_{0}}, \text { for some } p_{0} \in \mathcal{P}_{r}
$$

implies

$$
\lim _{n \rightarrow \infty} \frac{U\left(r^{n} z\right)}{\left(r^{n} z\right)^{\rho} \ell\left(r^{n} z\right)}=p(z) \quad \text { for each } z \in C_{p}, \text { for some } p \in \mathcal{P}_{r} .
$$

Conversely, if $u$ is ultimately nonincreasing then the reverse implication also holds, i.e. 2.27) implies 2.26. Moreover, $p=\mathrm{B}_{\rho} p_{0}$, in particular $p \in \mathcal{P}_{r, \rho}$ is continuous, and thus

$$
U(x) \sim x^{\rho} \ell(x) p(x) \quad \text { as } x \rightarrow \infty .
$$

For $\rho=0$ assume further that $\int_{0}^{\infty} u(y) \mathrm{d} y<\infty$. Then $\lim _{x \rightarrow \infty} U(x) / \ell(x)=\infty$, and $U \in \mathcal{S} \mathcal{V}_{\infty}$.

For continuous $p$ see [11, Lemma 3], [9, Theorem 6.8].

The corresponding result at 0 is the following.

Proposition 2.4. Let $U(x)=\int_{0}^{x} u(y) \mathrm{d} y$, where $u$ is ultimately monotone and locally integrable, $r>1, \rho>0$, and $\ell \in \mathcal{S} \mathcal{V}_{0}$. Then

$$
\lim _{n \rightarrow \infty} \frac{u\left(r^{-n} z\right)}{\left(r^{-n} z\right)^{\rho-1} \ell\left(r^{-n} z\right)}=p_{0}(z) \quad \text { for each } z \in C_{p_{0}}, \text { for some } p_{0} \in \mathcal{P}_{r}
$$

if and only if

$$
\lim _{n \rightarrow \infty} \frac{U\left(r^{-n} z\right)}{\left(r^{-n} z\right)^{\rho} \ell\left(r^{-n} z\right)}=p(z) \quad \text { for each } z \in C_{p}, \text { for some } p \in \mathcal{P}_{r} .
$$

Moreover, $p=\mathrm{B}_{\rho} p_{0}$, in particular $p$ is continuous, and thus

$$
U(x) \sim x^{\rho} \ell(x) p(x) \quad \text { as } x \downarrow 0 .
$$

\section{APPLICATIONS}

3.1. Tails of nonnegative random variables. Tail behavior of random variables is an important topic in probability theory, as the tail determines the asymptotic behavior of sums of iid and, under appropriate dependence conditions, non-iid random variables. When dealing with iid sums, the Laplace transform is a considerably simpler object than the distribution function. Moreover, for nonnegative random variables, the Laplace transform determines the distribution, in particular it also includes information about tail behavior. 
In this subsection we prove that the tail of a nonnegative random variable is regularly log-periodic if and only if the same holds for its Laplace transform at 0 . The corresponding result for regularly varying tails was proved by Bingham and Doney [7, Theorem A] (see also [8, Theorem 8.1.8]).

Let $X$ be a nonnegative random variable with distribution function $F$. If the moment condition $\mathbf{E} X^{m}<\infty$ holds then the Laplace transform

$$
\widehat{F}(s)=\int_{[0, \infty)} e^{-s x} \mathrm{~d} F(x)
$$

can be expanded in a Taylor series up to the term $s^{m}$ as

$$
\widehat{F}(s)=\sum_{k=0}^{m} \mu_{k} \frac{(-s)^{k}}{k !}+o\left(s^{m}\right) \quad \text { as } s \downarrow 0,
$$

where $\mu_{k}=\mathbf{E} X^{k}$. In order to compare the tail behavior of $X$ with the behavior of $\widehat{F}(s)$ as $s \downarrow 0$, we define the remainder $f_{m}$ of the Taylor expansion, and its derivative $g_{m}$ for $m \geqslant 0$ as

$$
\begin{aligned}
& f_{m}(s)=(-1)^{m+1}\left[\widehat{F}(s)-\sum_{k=0}^{m} \mu_{k} \frac{(-s)^{k}}{k !}\right], \\
& g_{m}(s)=\frac{\mathrm{d}^{m}}{\mathrm{~d} s^{m}} f_{m}(s)=\mu_{m}-(-1)^{m} \widehat{F}^{(m)}(s) .
\end{aligned}
$$

For $m=0$ we simply have $f_{0}(s)=g_{0}(s)=1-\widehat{F}(s)$.

THEOREM 3.1. Let $\ell \in \mathcal{S} \mathcal{V}_{\infty}, m \in\{0,1, \ldots\}, \alpha=m+\beta, \beta \in[0,1]$, and $\tilde{q}_{m}, q_{m}, p \in \mathcal{P}_{r}$. The following are equivalent:

$$
\begin{aligned}
& f_{m}(s) \sim s^{\alpha} \ell(1 / s) \tilde{q}_{m}(s) \quad \text { as } s \downarrow 0 ; \\
& g_{m}(s) \sim s^{\beta} \ell(1 / s) q_{m}(s) \quad \text { as } s \downarrow 0 ; \\
& \begin{cases}\lim _{n \rightarrow \infty} \ell\left(r^{n} z\right)^{-1} \int_{\left(r^{n} z, \infty\right)} y^{m} \mathrm{~d} F(y)=p(z), z \in C_{p}, & \beta=0, \\
\lim _{n \rightarrow \infty} \frac{\left(r^{n} z\right)^{\alpha}}{\ell\left(r^{n} z\right)} \bar{F}\left(r^{n} z\right)=p(z), z \in C_{p}, & \beta \in(0,1), \\
\lim _{n \rightarrow \infty} \ell\left(r^{n} z\right)^{-1} \int_{\left[0, r^{n} z\right]} y^{m+1} \mathrm{~d} F(y)=p(z), z \in C_{p}, & \beta=1 .\end{cases}
\end{aligned}
$$

If $\beta>0$, then (3.3)-(3.5) are further equivalent to

$$
(-1)^{m+1} \widehat{F}^{(m+1)}(s) \sim s^{\beta-1} \ell(1 / s) q_{m+1}(s) \quad \text { as } s \downarrow 0,
$$

and $q_{m+1}=\mathrm{B}_{\beta}^{-1} q_{m}$. 
Moreover, the relations between the functions involved are the following:

$$
\begin{aligned}
& q_{m}=\mathrm{B}_{\alpha-(m-1)}^{-1} \mathrm{~B}_{\alpha-(m-2)}^{-1} \ldots \mathrm{B}_{\alpha}^{-1} \widetilde{q}_{m}, \quad \beta \in[0,1], \quad q_{0}=\widetilde{q}_{0}, \\
& p_{0, m}=\mathrm{B}_{1-\beta}^{-1} \mathrm{~A}_{1-\beta}^{-1} q_{m}, \quad \beta \in(0,1), \\
& p=p_{0, m}-m \mathrm{~B}_{-m-\beta} p_{0, m}, p_{0, m}=p+m \mathrm{~B}_{-\beta} p, \quad \beta \in(0,1) .
\end{aligned}
$$

If $\beta \in\{0,1\}$, then necessarily $p(x) \equiv p>0, q_{m}(s) \equiv q_{m}>0$, and $p=q_{m}$.

Since $p(x)$ is constant for $\beta \in\{0,1\}$, by Lemma 2.1 the corresponding conditions in $\sqrt{3.5}$ are further equivalent to $\int_{(x, \infty)} y^{m} \mathrm{~d} F(y) \sim p \ell(x)$ and $\int_{[0, x]} y^{m+1} \mathrm{~d} F(y) \sim p \ell(x)$ as $x \rightarrow \infty$, respectively.

Proof of Theorem 3.1. We follow the proof of [8, Theorem 8.1.8].

First we show that $(3.3) \Leftrightarrow 3.4) \Leftrightarrow 3.6$. The equivalence of $(3.3)$ and $(3.4)$ follows from iterated application of Proposition 2.4. (Note that the derivatives of $f_{m}$ are monotone.) We find that $q_{m}=\mathrm{B}_{\alpha-(m-1)}^{-1} \mathrm{~B}_{\alpha-(m-2)}^{-1} \ldots \mathrm{B}_{\alpha}^{-1} \widetilde{q}_{m}$. Furthermore, for $\beta>0$ both 3.3 and 3.4 are equivalent to 3.6 , and $q_{m+1}=\mathrm{B}_{\beta}^{-1} q_{m}$.

The more difficult part is the equivalence 3.4$) \Leftrightarrow(3.5)$. Put

$$
U_{m}(x)=\int_{0}^{x} \int_{(t, \infty)} y^{m} \mathrm{~d} F(y) \mathrm{d} t,
$$

and note that by Fubini's theorem,

$$
\begin{aligned}
\widehat{U}_{m}(s) & =\int_{0}^{\infty} e^{-s x} \mathrm{~d} U_{m}(x)=\int_{0}^{\infty} \int_{(x, \infty)} e^{-s x} y^{m} \mathrm{~d} F(y) \mathrm{d} x \\
& =\frac{1}{s} \int_{[0, \infty)} y^{m}\left(1-e^{-s y}\right) \mathrm{d} F(y)=\frac{g_{m}(s)}{s} .
\end{aligned}
$$

Therefore (3.4) is equivalent to

$$
\widehat{U}_{m}(s) \sim s^{\beta-1} \ell(1 / s) q_{m}(s) \quad \text { as } s \downarrow 0 .
$$

For $\beta \in[0,1]$, by Theorem 2.1 with $\rho=1-\beta,(3.7)$ is further equivalent to

$$
\lim _{n \rightarrow \infty} \frac{U_{m}\left(r^{n} z\right)}{\left(r^{n} z\right)^{1-\beta} \ell\left(r^{n} z\right)}=p_{m}(z), \quad z \in C_{p_{m}},
$$

where $p_{m}=\mathrm{A}_{1-\beta}^{-1} q_{m}$ for $\beta \neq 1$, and $p_{m}=q_{m}$ for $\beta=1$.

First assume $\beta \in(0,1)$. By Theorems 2.2 and 2.3 with $\rho=1-\beta$, 3.8 holds if and only if

$$
\lim _{n \rightarrow \infty} \frac{u_{m}\left(r^{n} z\right)}{\left(r^{n} z\right)^{-\beta} \ell\left(r^{n} z\right)}=p_{0, m}(z), \quad z \in C_{p_{0, m}}
$$


where $u_{m}(x)=\int_{(x, \infty)} y^{m} \mathrm{~d} F(y)$ and $\mathrm{B}_{1-\beta} p_{0, m}=p_{m}$. Note that for $m=0$ this is exactly (3.5). Partial integration gives

$$
u_{m}(x)=x^{m} \bar{F}(x)+m \int_{x}^{\infty} y^{m-1} \bar{F}(y) \mathrm{d} y .
$$

Assume (3.5). Then applying Proposition 2.3 with $\rho=-\beta$ for the function $y^{m-1} \bar{F}(y)$ in the second term on the right-hand side of 3.10 , we obtain

$$
\lim _{n \rightarrow \infty} \frac{\left(r^{n} z\right)^{\alpha-m}}{\ell\left(r^{n} z\right)} \int_{r^{n} z}^{\infty} y^{m-1} \bar{F}(y) \mathrm{d} y=\mathrm{B}_{-\beta} p(z), \quad z \in C_{\mathrm{B}_{-\beta} p} .
$$

Thus (3.9) holds true with $p_{0, m}=p+m \mathrm{~B}_{-\beta} p$, so (3.4) follows.

Conversely, assume (3.4), which is equivalent to (3.9). Using Fubini's theorem we have

$$
x^{m} \int_{x}^{\infty} y^{-m-1} u_{m}(y) \mathrm{d} y=\int_{x}^{\infty} y^{m-1} \bar{F}(y) \mathrm{d} y,
$$

and therefore, by (3.10),

$$
x^{m} \bar{F}(x)=u_{m}(x)-m x^{m} \int_{x}^{\infty} y^{-m-1} u_{m}(y) \mathrm{d} y .
$$

Now, Proposition 2.3 with $\rho=-m-\beta$ shows that 3.9 implies

$$
\lim _{n \rightarrow \infty} \frac{\int_{r^{n} z}^{\infty} y^{-m-1} u_{m}(y) \mathrm{d} y}{\left(r^{n} z\right)^{-m-\beta} \ell\left(r^{n} z\right)}=\mathrm{B}_{-m-\beta} p_{0, m}(z) .
$$

Thus, if (3.9) holds, then by 3.11,

$$
\lim _{n \rightarrow \infty} \frac{\left(r^{n} z\right)^{m+\beta}}{\ell\left(r^{n} z\right)} \bar{F}\left(r^{n} z\right)=p_{0, m}(z)-m \mathrm{~B}_{-m-\beta} p_{0, m}(z), \quad z \in C_{p_{0, m}},
$$

which is exactly (3.5).

For $\beta=0$ conditions (3.8) and (3.9) are still equivalent. If (3.9) holds, then the monotonicity of $u$ forces that $p_{0, m}$ is constant, and (3.5) follows with $p=p_{0, m}$. The converse is obvious.

For $\beta=1$ note that $(-1)^{m+1} \widehat{F}^{(m+1)}(s)$ is the Laplace-Stieltjes transform of $\int_{[0, x]} y^{m+1} \mathrm{~d} F(y)$. Therefore, by Theorem 2.1. 3.5. and 3.6 are equivalent, and $q_{m+1}=p$.

We spell out this result in the most important special case, when $m=0$. In this case $f_{0}(s)=g_{0}(s)=1-\widehat{F}(s)$. 
COROllary 3.1. Let $\ell \in \mathcal{S} \mathcal{V}_{\infty}, \alpha \in[0,1]$, and $q_{0}, p \in \mathcal{P}_{r}$. The following are equivalent:

$$
\begin{aligned}
& 1-\widehat{F}(s) \sim s^{\alpha} \ell(1 / s) q_{0}(s) \quad \text { as } s \downarrow 0 ; \\
& \begin{cases}\lim _{n \rightarrow \infty} \frac{\left(r^{n} z\right)^{\alpha}}{\ell\left(r^{n} z\right)} \bar{F}\left(r^{n} z\right)=p(z), z \in C_{p}, & \alpha \in[0,1), \\
\lim _{n \rightarrow \infty} \ell\left(r^{n} z\right)^{-1} \int_{\left[0, r^{n} z\right]} y \mathrm{~d} F(y)=p(z), z \in C_{p}, & \alpha=1 .\end{cases}
\end{aligned}
$$

If $\alpha>0$, then 3.13 and 3.14 are further equivalent to

$$
-\widehat{F}^{\prime}(s) \sim s^{\alpha-1} \ell(1 / s) q_{1}(s) \quad \text { as } s \downarrow 0,
$$

and $q_{1}=\mathrm{B}_{\alpha}^{-1} q_{0}$.

Moreover, $p=\mathrm{B}_{1-\alpha}^{-1} \mathrm{~A}_{1-\alpha}^{-1} q_{0}$ if $\alpha \in(0,1)$. If $\alpha \in\{0,1\}$, then necessarily $p(x) \equiv p>0, q_{0}(s) \equiv q_{0}>0$, and $p=q_{0}$.

EXAMPLE 3.1 (Generalized St. Petersburg games). The random variable $X$ has the generalized St. Petersburg distribution with parameter $\alpha \in(0,1]$ (and $p=q=1 / 2)$ if $\mathbf{P}\left\{X=2^{n / \alpha}\right\}=2^{-n}, n=1,2, \ldots$ The tail of the distribution function is

$$
\bar{F}(x)=\mathbf{P}\{X>x\}=\frac{2^{\left\{\alpha \log _{2} x\right\}}}{x^{\alpha}}, \quad x \geqslant 2^{1 / \alpha},
$$

where $\{x\}$ stands for the fractional part of $x$. For generalized St. Petersburg games we refer to Csörgó [14], Berkes, Györfi, and Kevei [3], and the references therein.

With the notation of Corollary 3.1. for $\alpha<1$ we have $r=2^{1 / \alpha}, p(z) \equiv$ $2^{\left\{\alpha \log _{2} z\right\}}$, and $\ell(x) \equiv 1$, while if $\alpha=1$ then $r=2, p(z) \equiv 1$, and $\ell(x)=\log _{2} x$. In this special case of the Laplace transform

$$
\widehat{F}(s)=\sum_{n=1}^{\infty} e^{-2^{n / \alpha}} s 2^{-n}
$$

explicit computation shows that

$$
1-\widehat{F}(s) \sim s^{\alpha} \sum_{m=-\infty}^{\infty}\left(1-\exp \left[-2^{\frac{m-\left\{-\alpha \log _{2} s\right\}}{\alpha}}\right]\right) 2^{-m+\left\{-\alpha \log _{2} s\right\}}=: s^{\alpha} q_{0}(s)
$$

as $s \downarrow 0$ whenever $\alpha<1$, and $1-\widehat{F}(s) \sim s \log _{2} s^{-1}$ as $s \downarrow 0$ for $\alpha=1$. This is exactly the statement of Corollary 3.1. A somewhat lengthy but straightforward calculation shows that $q_{0}=A_{1-\alpha} B_{1-\alpha} p$ for $\alpha<1$.

3.2. Fixed points of smoothing transforms. Let $T=\left(T_{i}\right)_{i \in \mathbb{N}}$ be a sequence of nonnegative random variables; it can be finite or infinite, dependent or independent. 
A random variable $X$, or its distribution, is the fixed point of the homogeneous smoothing transform corresponding to $T$ if

$$
X \stackrel{\mathcal{D}}{=} \sum_{i \geqslant 1} X_{i} T_{i}
$$

where on the right-hand side $X_{1}, X_{2}, \ldots$ are iid copies of $X$, and they are independent of $T$.

The theory of smoothing transforms goes back to Mandelbrot [31]. Existence and behavior of the solution of equations of type (3.16) was investigated by Durrett and Liggett [16], Guivarc'h [22], Liu [30], Biggins and Kyprianou [5], and Alsmeyer, Biggins, and Meiners [1], to mention but a few. For applications and references we refer to Section 5.2 in the monograph [12] by Buraczewski, Damek, and Mikosch.

Most of the results on the tail behavior of the solution provide conditions which imply exact power-law tail. We are aware of very few exceptions. Theorem 2.2 in [30] states that in the arithmetic case, under appropriate conditions there is an $\alpha>0$ such that

$$
0<\liminf _{x \rightarrow \infty} x^{\alpha} \mathbf{P}\{X>x\} \leqslant \limsup _{x \rightarrow \infty} x^{\alpha} \mathbf{P}\{X>x\}<\infty .
$$

Guivarc'h [22, p. 268] noted without proof that in the arithmetic case under appropriate conditions on the tail of $X$, the solution of 3.16) behaves like $p(x) x^{-\alpha}$ for some $p \in \mathcal{P}_{r, \alpha}$.

To prove tail asymptotics for the solutions of general stochastic fixed point equations, implicit renewal theory is a powerful technique. The idea goes back to Grincevičius [20] who determined the tail behavior of the solution of the perpetuity equation (1.2). The method was extended and developed further by Goldie [19] to determine the tail of more general stochastic fixed point equations. For the nonhomogeneous smoothing transform the method was worked out by Jelenković and Olvera-Cravioto [26] both in the arithmetic and in the nonarithmetic case. Implicit renewal theory typically works in situations where the properties of the solution are determined by the second root of the equation and $m(\theta)=1$, where $m(\theta)$ is a convex function related to the fixed point equation. (For the perpetuity equation (1.2) and $m(\theta)=\mathbf{E} A^{\theta}$, for the smoothing transform see below.) In the following example in the setup of [1], the behavior of the solution is governed by the root $\alpha$ of the equation $m(\theta)=1$ for which $m^{\prime}(\alpha)<0$ (see conditions (iii) and (iv)), so implicit renewal theory does not work.

In order to state the main result in [1] we need some further definitions and assumptions. Let $N=\sum_{i} I\left(T_{i}>0\right)$ denote the number of positive terms on the right-hand side of 3.16 , and assume without loss of generality that $N=$ $\sup \left\{i \geqslant 1: I\left(T_{i}\right)>0\right\}$, where $I(A)$ stands for the indicator of the set $A$. Put $m(\theta)=\mathbf{E} \sum_{i=1}^{N} T_{i}^{\theta}$. Assume that 
(i) $\mathbf{P}\left\{T \in\{0,1\}^{\mathbb{N}}\right\}<1$;

(ii) $\mathbf{E} N>1$;

(iii) there exists $\alpha \in(0,1]$ such that $1=m(\alpha)<m(\beta)$ for $\beta \in[0, \alpha)$;

(iv) either there is a $\theta \in[0, \alpha)$ such that $m(\theta)<\infty$, or

$$
\mathbf{E} \sum_{i \geqslant 1} T_{i}^{\alpha} \log T_{i} \in(-\infty, 0) \quad \text { and } \quad \mathbf{E}\left(\sum_{i \geqslant 1} T_{i}^{\alpha}\right) \log _{+} \sum_{i \geqslant 1} T_{i}^{\alpha}<\infty
$$

(v) there exists a nonnegative random variable $W$, not identically 0 , such that

$$
W \stackrel{\mathcal{D}}{=} \sum_{i \geqslant 1} T_{i}^{\alpha} W_{i}
$$

where $W_{1}, W_{2}, \ldots$ are iid copies of $W$ that are independent of $T$, and $T$ has the same distribution as in 3.16;

(vi) the positive elements of $T$ are concentrated on $r^{\mathbb{Z}}$ for some $r>1$, and $r$ is the smallest such number.

Under the above assumptions, in [1, Corollary 2.3] it was showed that the Laplace transform $\varphi$ of the solution of the fixed point equation 3.16 has the form

$$
\varphi(t)=\psi\left(h(t) t^{\alpha}\right), \quad t \geqslant 0,
$$

where $\alpha \in(0,1], h$ is a logarithmically $r$-periodic function such that $h(t) t^{\alpha}$ is a Bernstein function, i.e. its derivative is completely monotone, and $\psi$ is the Laplace transform of the random variable $W$ in (v) such that $(1-\psi(t)) t^{-1}$ is slowly varying at 0 .

The tail behavior of the solutions was not discussed in [1]. Theorem 3.1, in particular Corollary 3.1, allows us to determine it. Namely, (3.17) and the slow variation of $(1-\psi(t)) t^{-1}$ imply

$$
1-\varphi(t)=\frac{1-\psi\left(h(t) t^{\alpha}\right)}{h(t) t^{\alpha}} h(t) t^{\alpha}=: \ell\left(\frac{1}{h(t) t^{\alpha}}\right) h(t) t^{\alpha}=\tilde{\ell}(1 / t) h(t) t^{\alpha}
$$

for some function $\ell$ slowly varying at infinity. Note that $\ell \in \mathcal{S} \mathcal{V}_{\infty}$ implies $\tilde{\ell}(x)=$ $\ell\left(x^{\alpha} h(x)\right) \in \mathcal{S} \mathcal{V}_{\infty}$. We can apply Corollary 3.1. Noting that $\ell\left(x^{\alpha} h(x)\right) \sim \ell\left(x^{\alpha}\right)$ as $x \rightarrow \infty$, we obtain the following.

COROllary 3.2. Assume (i)-(vi). If $\alpha<1$, then for the solution of 3.16 we have

$$
\lim _{n \rightarrow \infty} \frac{\left(r^{n} z\right)^{\alpha}}{\ell\left(r^{\alpha n}\right)} \mathbf{P}\left\{X>r^{n} z\right\}=p(z), \quad z \in C_{p},
$$

where $p=\mathrm{B}_{1-\alpha}^{-1} \mathrm{~A}_{1-\alpha}^{-1} h$. If $\alpha=1$, then $h(t) \equiv h$ is necessarily a constant, and for the truncated first moment we have

$$
\mathbf{E}(X I(X \leqslant x)) \sim h \ell(x) \quad \text { as } x \rightarrow \infty .
$$


3.3. Semistable laws. Logarithmically periodic functions and regularly log-periodic functions naturally arise in the analysis of semistable distributions. The class of semistable laws, introduced by Paul Lévy, is an important subclass of infinitely divisible laws. This class includes the stable laws and those infinitely divisible distributions which have no normal component and whose Lévy measure $\mu$ in the Lévy-Khinchin representation satisfies

$$
\mu((x, \infty))=x^{-\alpha} p_{+}(x), \quad \mu((-\infty,-x))=x^{-\alpha} p_{-}(x), \quad x>0,
$$

where $\alpha \in(0,2), r>1$, and $p_{+}, p_{-} \in \mathcal{P}_{r,-\alpha} \cup\{0\}$ (here 0 is the identically zero function), with $p_{+}, p_{-}$not both identically 0 . For properties, characterization, applications and some history of semistable laws we refer to the monograph by Meerschaert and Scheffler [32], to the papers by Megyesi [33] and by Huillet, Porzio, and Ben Alaya [24], and to the references therein. For a more recent account of semistability see Chaudhuri and Pipiras [13]. We note that in the characterization of the domain of geometric partial attraction regularly log-periodic functions play an important role: see Grinevich and Khokhlov [21], and Megyesi [33].

Although there has been much interest in semistable laws in the last 50 years, the tail behavior was determined completely only in 2012 by Watanabe and Yamamuro [38]; for partial results for nonnegative semistable distributions see [24, p. 357] for $p$ continuous, and Shimura and Watanabe [34, Theorem 1.3] for general $p$. We re-prove some of the results in [38], emphasizing that more precise and more general results were shown there. In particular, we restrict ourselves to nonnegative semistable laws, since the technique developed in this paper works only for one-sided laws.

The Laplace transform of a nonnegative semistable random variable $W$ has the form

$$
\mathbf{E} e^{-s W}=\exp \left\{-a s-\int_{(0, \infty)}\left(1-e^{-s y}\right) \mu(\mathrm{d} y)\right\}
$$

where $a \geqslant 0$, and $\mu$ is a Lévy measure such that $\bar{\mu}(x)=p(x) x^{-\alpha}$ with $p \in \mathcal{P}_{r,-\alpha}$, $\alpha \in(0,1)$, and $\bar{\mu}(x)=\mu((x, \infty))$ for $x>0$. Integration by parts gives

$$
\int_{(0, \infty)}\left(1-e^{-s y}\right) \mu(\mathrm{d} y)=\int_{0}^{\infty} e^{-s y} s \bar{\mu}(y) \mathrm{d} y=s \widehat{U}(s),
$$

where

$$
U(x)=\int_{0}^{x} \bar{\mu}(y) \mathrm{d} y=x^{1-\alpha} \mathrm{B}_{1-\alpha} p(x) .
$$

From Theorem 2.1 we have $\widehat{U}(s) \sim s^{\alpha-1} q(s)$ as $s \downarrow 0$ with $q=\mathrm{A}_{1-\alpha} \mathrm{B}_{1-\alpha} p$. Thus, (3.18) gives

$$
1-\mathbf{E} e^{-s W} \sim a s+\int_{(0, \infty)}\left(1-e^{-s y}\right) \mu(\mathrm{d} y) \sim s^{\alpha} q(s) \quad \text { as } s \downarrow 0 .
$$


Corollary 3.1 implies $\lim _{n \rightarrow \infty}\left(r^{n} z\right)^{\alpha} \mathbf{P}\left\{W>r^{n} z\right\}=p(z)$ for each $z \in C_{p}$, or, which is the same,

$$
\lim _{n \rightarrow \infty} r^{n \alpha} \mathbf{P}\left\{W>r^{n} z\right\}=\bar{\mu}(z) \quad \text { for each } z \in C_{p} .
$$

This is stated in [38, Theorem 1]. However, the limit above is determined there for any $z>0$. The proof in [38] exploits the fact that the distribution of $W$ is infinitely divisible and relies on the heavy machinery regarding the ratio of the tail of the distribution function and the tail of the Lévy measure. Our method only uses the asymptotic behavior of the Laplace transform at zero, which is basically the same for any distribution from the domain of geometric partial attraction of the semistable law $W$. As described in Example 2.2, it is possible to change the distribution function in such a way that the limit in (3.19) does not change for $z \in C_{p}$, but does not even exist for $z \notin C_{p}$. Therefore, based only on Tauberian theorems, the limit in (3.19) for $z \notin C_{p}$ cannot be determined.

3.4. Supercritical Galton-Watson processes. Consider a Galton-Watson process $\left(Z_{n}\right)_{n \in \mathbb{N}}, Z_{0}=1$, with offspring generating function $f(s)=\mathbf{E} s^{Z_{1}}$, and offspring mean $\mu=\mathbf{E} Z_{1} \in(1, \infty)$. Let $q \in[0,1)$ denote the extinction probability, i.e. the smallest root of $f(s)=s$ in $[0,1]$. Denote by $f_{n}$ the $n$-fold iterate of $f$, which is the generating function of $Z_{n}$. For a general theory of branching processes see Athreya and Ney [2].

Further assume $\mathbf{E} Z_{1} \log Z_{1}<\infty$, which ensures that

$$
Z_{n} / \mu^{n} \rightarrow W \quad \text { as } n \rightarrow \infty \text { a.s. }
$$

with $\mathbf{E} W=1$. The Laplace transform of $W, \varphi(t)=\mathbf{E} e^{-t W}, t \geqslant 0$, satisfies the Poincaré functional equation

$$
\varphi(\mu t)=f(\varphi(t))
$$

The latter equation always has a unique solution (up to scaling), which is the Laplace transform of a distribution. However, the law of $W$ can be determined explicitly only in very few special cases. Therefore, it is important to obtain asymptotic behavior of the tail probabilities. Assume that we are in the Schröder case, that is, $\gamma=f^{\prime}(q)>0$. Put $\rho=-\log \gamma / \log \mu$. Harris [23, Theorem 3.3] proved that

$$
\varphi(s)-q \sim K(s) / s^{\rho} \quad \text { as } s \rightarrow \infty,
$$

where $K$ is a logarithmically periodic function with period $\mu$. Note that the limit distribution in (3.20) puts mass $q$ at 0 , therefore $\lim _{s \rightarrow \infty} \varphi(s)=q$. From a version of Theorem 2.1 with $n \rightarrow-\infty$ in (2.7) and $s \rightarrow \infty$ in $(2.8)$, it follows for the distribution function

$$
G(x)=\mathbf{P}\{W \leqslant x\}=\mathbf{P}\{W=0\}+\mathbf{P}\{0<W \leqslant x\}=q+\mathbf{P}\{0<W \leqslant x\}
$$


that

$$
\lim _{n \rightarrow \infty}\left[G\left(r^{-n} z\right)-q\right]\left(r^{-n} z\right)^{-\rho}=p(z)
$$

with $p=\mathrm{A}_{\rho}^{-1} K$, where $K$ comes from (3.22).

Much more is known about the tail probabilities $\mathbf{P}\{0<W \leqslant x\}$ as $x \downarrow 0$. The distribution of the limiting random variable $W$ is absolutely continuous on $(0, \infty)$ [2, Theorem I.10.4, Corollary I.12.1], and the density function $g$ is continuous (see Dubuc [15]). Therefore, (3.23) can be written as

$$
\lim _{n \rightarrow \infty} \frac{\int_{0}^{r^{-n} z} g(y) \mathrm{d} y}{\left(r^{-n} z\right)^{\rho}}=p(z),
$$

which suggests by Proposition 2.4 that

$$
g(x) \sim x^{\rho-1} p_{0}(x) \quad \text { as } x \downarrow 0,
$$

where $p_{0}=\mathrm{B}_{\rho}^{-1} p$. Note that this argument is not a proof, as the monotonicity of $g$ is missing, which is a crucial condition in Proposition 2.4. Based on the functional equation (3.21), Biggins and Bingham [4, Theorem 4] proved that 3.25$]$ is indeed correct, where $p_{0}$ is a continuous, positive, logarithmically periodic function with period $\mu$. For further results on tail asymptotics of $W$ we refer to Bingham [6], Biggins and Bingham [4], and to the more recent papers by Fleischmann and Wachtel [18] and by Wachtel, Denisov, and Korshunov [37].

Acknowledgments. I am grateful to the anonymous referees for their remarks and suggestions which improved the paper.

\section{REFERENCES}

[1] G. Alsmeyer, J. D. Biggins, and M. Meiners, The functional equation of the smoothing transform, Ann. Probab. 40 (2012), 2069-2105.

[2] K. B. Athreya and P. E. Ney, Branching Processes, Springer, New York, 1972.

[3] I. Berkes, L. Györfi, and P. Kevei, Tail probabilities of St. Petersburg sums, trimmed sums, and their limit, J. Theoret. Probab. 30 (2017), 1104-1129.

[4] J. D. Biggins and N. H. Bingham, Large deviations in the supercritical branching process, Adv. Appl. Probab. 25 (1993), 757-772.

[5] J. D. Biggins and A. E. Kyprianou, Fixed points of the smoothing transform: the boundary case, Electron. J. Probab. 10 (2005), 609-631.

[6] N. H. Bingham, On the limit of a supercritical branching process, J. Appl. Probab. 25A (1988) (special volume), 215-228.

[7] N. H. Bingham and R. A. Doney, Asymptotic properties of supercritical branching processes. I. The Galton-Watson process, Adv. Appl. Probab. 6 (1974), 711-731.

[8] N. H. Bingham, C. M. Goldie, and J. L. Teugels, Regular Variation, Encyclopedia Math. Appl. 27, Cambridge Univ. Press, Cambridge, 1989.

[9] V. V. Buldygin, K.-H. Indlekofer, O. I. Klesov, and J. G. Steinebach, Pseudo-Regularly Varying Functions and Generalized Renewal Processes, Springer, 2018. 
[10] V. V. Buldigin and V. V. Pavlenkov, A generalization of Karamata's theorem on the asymptotic behavior of integrals, Teor. Ĭmovīr. Mat. Statist. 81 (2009), 13-24 (in Ukrainian); English transl.: Theory Probab. Math. Statist. 81 (2010), 15-26.

[11] V. V. Buldygin and V. V. Pavlenkov, Karamata theorem for regularly log-periodic functions, Ukrainian Math. J. 64 (2013), 1635-1657.

[12] D. Buraczewski, E. Damek, and T. Mikosch, Stochastic Models with Power-Law Tails. The Equation $X=A X+B$, Springer, 2016.

[13] R. Chaudhuri and V. Pipiras, Non-Gaussian semi-stable laws arising in sampling of finite point processes, Bernoulli 22 (2016), 1055-1092.

[14] S. Csörgő, Rates of merge in generalized St. Petersburg games, Acta Sci. Math. (Szeged) 68 (2002), 815-847.

[15] S. Dubuc, Problèmes relatifs à l'itération de fonctions suggérés par les processus en cascade, Ann. Inst. Fourier (Grenoble) 21 (1971), no. 1, 171-251.

[16] R. Durrett and T. M. Liggett, Fixed points of the smoothing transformation, Z. Wahrsch. Verw. Gebiete 64 (1983), 275-301.

[17] W. Feller, On the classical Tauberian theorems, Arch. Math. (Basel) 14 (1963), 317-322.

[18] K. Fleischmann and V. Wachtel, On the left tail asymptotics for the limit law of supercritical Galton-Watson processes in the Böttcher case, Ann. Inst. H. Poincaré Probab. Statist. 45 (2009), 201-225.

[19] C. M. Goldie, Implicit renewal theory and tails of solutions of random equations, Ann. Appl. Probab. 1 (1991), 126-166.

[20] A. K. Grincevičius, One limit distribution for a random walk on the line, Lithuanian Math. J. 15 (1975), 580-589.

[21] I. V. Grinevich and Y. S. Khokhlov, Domains of attraction of semistable laws, Teor. Veroyatnost. i Primenen. 40 (1995), 417-422 (in Russian); English transl.: Theory Probab. Appl. 40 (1995), 361-366.

[22] Y. Guivarc'h, Sur une extension de la notion de loi semi-stable, Ann. Inst. H. Poincaré Probab. Statist. 26 (1990), 261-285.

[23] T. E. Harris, Branching processes, Ann. Math. Statist. 19 (1948), 474-494.

[24] T. Huillet, A. Porzio, and M. B. Alaya, On Lévy stable and semistable distributions, Fractals 9 (2001), 347-364.

[25] A. Iksanov, Renewal Theory for Perturbed Random Walks and Similar Processes, Springer, 2016.

[26] P. R. Jelenković and M. Olvera-Cravioto, Implicit renewal theorem for trees with general weights, Stochastic Process. Appl. 122 (2012), 3209-3238.

[27] P. Kevei, Implicit renewal theory in the arithmetic case, J. Appl. Probab. 54 (2017), 732-749.

[28] B. I. Korenblyum, On the asymptotic behavior of Laplace integrals near the boundary of a region of convergence, Dokl. Akad. Nauk SSSR (N.S.) 104 (1955), 173-176 (in Russian).

[29] K.-S. Lau and C. R. Rao, Integrated Cauchy functional equation and characterizations of the exponential law, Sankhyā Ser. A 44 (1982), 72-90.

[30] Q. Liu, On generalized multiplicative cascades, Stochastic Process. Appl. 86 (2000), $263-286$.

[31] B. Mandelbrot, Multiplications aléatoires itérées et distributions invariantes par moyenne pondérée aléatoire, C. R. Acad. Sci. Paris Sér. A 278 (1974), 289-292.

[32] M. M. Meerschaert and H.-P. Scheffler, Limit Distributions for Sums of Independent Random Vectors, Wiley, New York, 2001.

[33] Z. Megyesi, A probabilistic approach to semistable laws and their domains of partial attraction, Acta Sci. Math. (Szeged) 66 (2000), 403-434.

[34] T. Shimura and T. Watanabe, Infinite divisibility and generalized subexponentiality, Bernoulli 11 (2005), 445-469.

[35] D. Sornette, Discrete-scale invariance and complex dimensions, Phys. Rep. 297 (1998), 239270. 
[36] U. Stadtmüller and R. Trautner, Tauberian theorems for Laplace transforms, J. Reine Angew. Math. 311/312 (1979), 283-290.

[37] V. I. Vakhtel', D. E. Denisov, and D. A. Korshunov, On the asymptotics of the tail of distribution of a supercritical Galton-Watson process in the case of heavy tails, Trudy Mat. Inst. Steklova 282 (2013), 288-314.

[38] T. Watanabe and K. Yamamuro, Tail behaviors of semi-stable distributions, J. Math. Anal. Appl. 393 (2012), 108-121.

\section{Péter Kevei}

MTA-SZTE Analysis and Stochastics Research Group

Bolyai Institute, Aradi vértanúk tere 1

6720 Szeged, Hungary

E-mail: kevei@math.u-szeged.hu 